\title{
Stereological Analysis of Polycrystalline Microstructure
}

\author{
James H. Steele Jr. Ph.D. Consultant, 2019 Nova Road, Pine, CO 80470
}

Stereological analysis of polycrystalline microstructure can be carried out using the physical disector [1], wherein closely spaced serial sections are analyzed for the appearance of new grains, faces, and triple junctions. A number of results of serial section analyses have been reported for the topological properties of polycrystals $[2,3,4]$. The topological properties include the number of grains, facets, triple junctions, and quadruple points present in the "statistical honeycomb" [5]. These topological properties also provide estimates of the average grain and facet shape, which are related by the famous Coxeter equation [5], $\mathbf{F}=\mathbf{1 2} /(\mathbf{6}-\mathbf{p})$

where $\mathbf{F}$ is the average number of faces per grain, and $\mathbf{p}$ is the average number of edges per facet. Coxeter also demonstrated that $\mathbf{p}$ should be 5.104, and therefore $\mathbf{F}$ should be 13.40 for cell structures

with facet angles at the vertices of $\cos ^{-1}(-1 / 3)\left(\right.$ i.e. $\left.109.5^{\circ}\right)$, which is required for a balance of surface tension forces within the boundary network.

Data from a series of serial sections through an $\mathrm{Al}-1 \% \mathrm{Mg}$ alloy structure are presented in Fig. 1, which shows optical micrographs of a pair of adjacent serial sections with the appearance of two new grains circled for identification. The cumulative counts of the number of features are shown as Fig. 2 for the 72 sections analyzed. The rules used for counting features intersecting the field boundaries were that those features intersecting the sides of the sectioned volume were counted as $1 / 2$, and those intersecting the corners as $1 / 4$. The number of features counted is thus called a cumulative effective number. The slope of the linear least squares fit of the straight line provides an estimate of the number per unit volume $\left(\mathrm{N}_{\mathbf{V}}\right)$. The coefficient of variation can be obtained from linear least squares fit of the data, although these estimates are not reported in this paper. Table I gives a listing of the estimates of the number of features per unit volume, and the average shapes obtained from these data.

Standard stereological measurements on 2-d sections can also provide estimates of the number of grains per unit volume, if a model for the 3-d microstructure of the polycrystalline aggregate is used. One of the classic models is the generalized Johnson-Mehl model described by Miles [6], which gives an average shape of $\mathbf{F}=13.3$. This model allows the estimated number of grains $\left(\mathbf{N}_{\mathbf{V}}\right)$ to be calculated from the average number of sections per unit area $\left(\mathbf{N}_{\mathbf{A}}\right)$ as, $\mathbf{N}_{\mathbf{A}}=1.258\left(\mathbf{N}_{\mathbf{V}}\right)^{\mathbf{2} / 3}$ (2)

References

[1] D.C. Sterio, J. Microsc. 134 (1984) 127.

[2] J.H. Steele Jr., Stereology, Elias (Ed.), Springer Verlag, N.Y. (1967) 74.

[3] R.T. DeHoff and G. Liu, Met. Trans. A 16 (1985) 2007.

[4] F.N. Rhines, et al., Met. Trans. 5 (1974) 413. 
[5] H.S.M. Coxeter, Ill. J. Math. 2 (1958) 746.

[6] R.E. Miles, Suppl. Adv. Appl. Prob. (1972) 243.

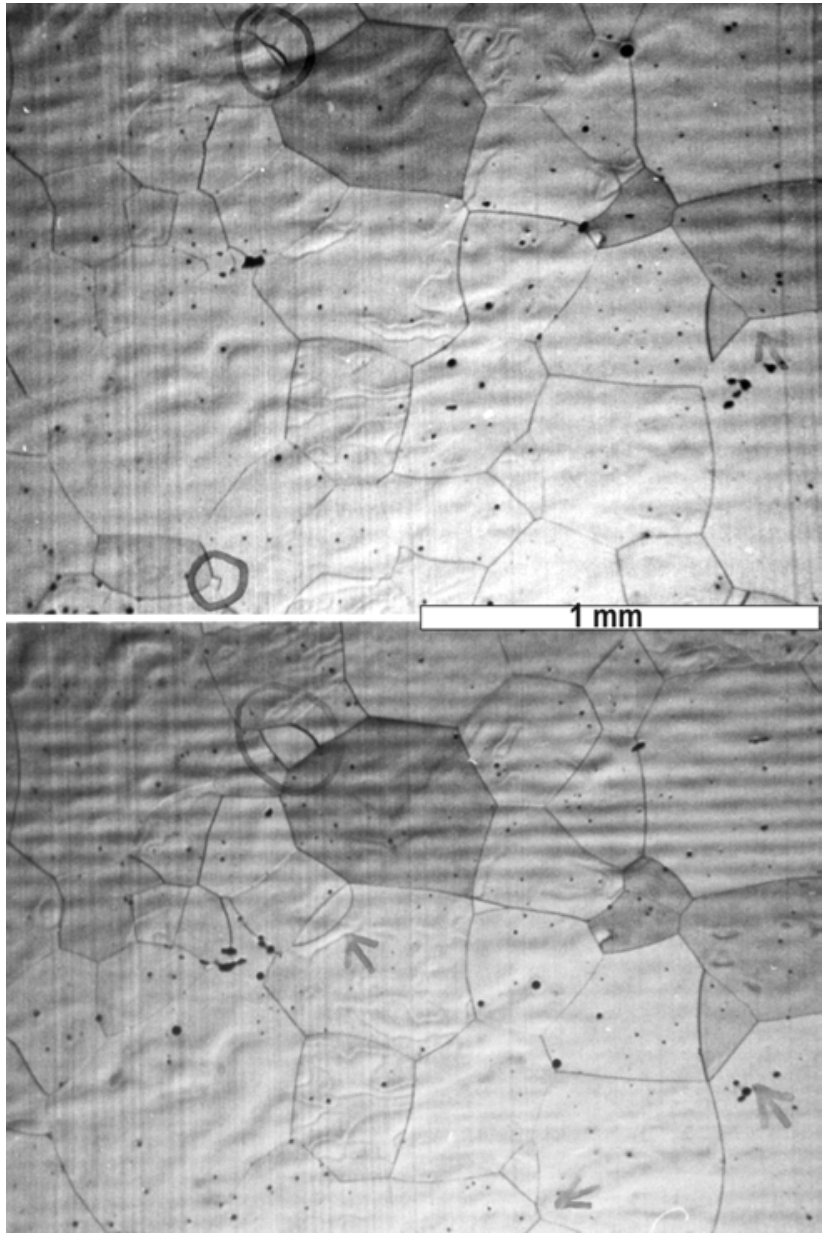

Fig. 1 Optical micrographs of a pair of serial sections.

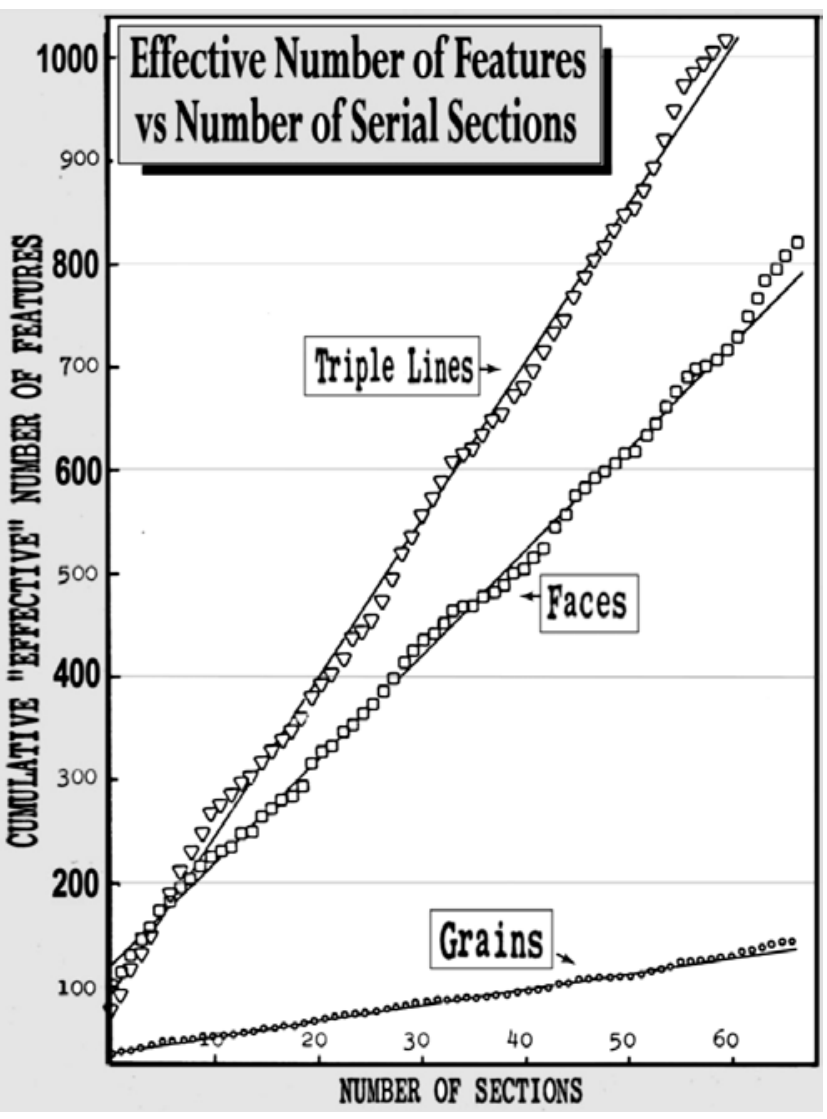

Fig. 2 Plot of cumulative number of features.

Table 1 Results from 2-d measurements and serial section analysis.

\begin{tabular}{|l|l|}
\hline Grains per Unit Volume & $49.5 \mathrm{~mm}^{-3}$ \\
\hline Facets per Unit Volume & $344 \mathrm{~mm}^{-3}$ \\
\hline Triple Junctions per Unit Volume & $524 \mathrm{~mm}^{-3}$ \\
\hline $\mathbf{F}=$ Average Grain Shape & 13.9 faces/grain \\
\hline $\mathbf{S}_{\mathbf{V}}$ from 2-d measurement & $8.25 \mathrm{~mm}^{2} / \mathrm{mm}^{3}$ \\
\hline $\mathbf{N}_{\mathbf{A}}$ from 2-d measurement & $18.3 \mathrm{~mm}^{-2}$ \\
\hline
\end{tabular}


https://doi.org/10.1017/S1431927603443687 Published online by Cambridge University Press 